\title{
Consideration of intelligent applications to support diabetic patients: A scoping review for nutrition mobile phone apps
}

\author{
Zahra Koohmareh, ${ }^{1}$ Amir Jamshidnezhad, ${ }^{1}$ and Ali Pazahr ${ }^{2}$
}

\author{
'Department of Health Information Technology, Faculty of Allied Medical Sciences, Ahvaz Jundishapur University of Medical Sciences, Ahvaz, Iran \\ 2Department of Computer Engineering, Islamic Azad University, Ahvaz branch, Ahvaz, Iran \\ Correspondence to: Amir Jamshidnezhad, Ahvaz Jundishapur University of Medical Sciences, Iran (email: Jamshidnejad-a@ajums.ac.ir). \\ (Submitted: 09 September 2019 - Revised version received: 04 October 2019 - Accepted: 22 October 2019 - Published online: 26 February 2020)
}

\begin{abstract}
Objectives The main objective is to identify the functionality of smart applications in mobile platform for controlling, teaching, and healthy lifestyles in the field of nutrition for diabetic patients.

Methods Current review was conducted on mobile platform to consider the functionalities of available applications to support the diabetic patients with the intelligent tools. The results of inclusion criteria (such as nutrition, weight control, blood pressure, education, and interactive analysis tools) were evaluated and organized in the form of a scoping review. All applications in the systematic search were independent of both operating systems. The search samples were found in May 2018 by experts in medical informatics.

Results 273 potential applications were identified according to the results of the inclusion criteria with evidence-based strategies and systematic evaluations to find the apps for diabetic patients. Approximately, 29\% of apps in both stores (79/273) met primary assessment criteria. Of these, $40 \%$ of apps (32/79) excluded from more consideration due to lack in most criteria of this study. The remaining apps are described as follows: $8 \%$ of total apps (22/273) and 4\% of the apps (11/273) met general criteria and all criteria (including general and special) in the Google play store, respectively. Only 2 apps met general criteria with the Apple iOS while none of them covered with special criteria according to the objectives of this study.

Conclusion There are various applications for control, education, and self-care in diabetes. Google is an acceptable operating system for designers interested in examining interactive apps and techniques in disease control, education, and healthy lifestyle programs.

Keywords mobile health, smart apps, diet, diabetes, self care
\end{abstract}

\section{Introduction}

Today, mobile phone has become a technology of modern communication in the social and individual life of human beings. Smart phone is an integral part of the daily activities and connections of human beings. The influence of mobile applications in various aspects of life is a universal phenomenon. ${ }^{1}$ Today, the use of mobile phones and their effects on our daily life, and particularly its effectiveness in the health care, is dramatically rising. ${ }^{2,3}$ Healthcare applications have made the smartphones as a useful tool in the traditional medicine for self-care and clinical interactions. Also, mobile phones have the significant role in education and monitoring of patients. ${ }^{4}$ Mobile health apps are widely used to diagnose chronic diseases such as cardiovascular and diabetes disease. ${ }^{5}$ Moreover, those have focused on interventional research of specific medical issues such as: skin cancer, ${ }^{6}$ weight management, ${ }^{7}$ and cardiovascular. ${ }^{8}$ These apps are not limited to diagnosis and evaluation of the patient's condition, but also include beyond the clinical aspects such as patient appointment, counseling, patient management, and medical training. ${ }^{9}$ The main operating systems for smartphones are Google Android and iOS Apple. Apple iTunes and Google Play stores offer many applications for mobile health. ${ }^{10}$

Intelligent methods as the learning-based interactive models have been introduced in medical fields such as stroke imaging, ${ }^{11,12}$ medical imaging, ${ }^{13}$ medical decision support, ${ }^{14}$ and medical data analysis. ${ }^{15}$ Patients with stroke can receive medical care information by the interactive models, which provide them with the prognosis and required treatments. ${ }^{11}$ Artificial intelligence is becoming more advanced in interventional applications such as weight control and daily activities.
Those apps with cognitive and scientific models can enhance human performance. ${ }^{16}$ The aim of this review research is to systematic investigation of mobile smart applications to provide the digital methods for monitoring the nutritional health of diabetic patients through self-care education and scientific consideration. The outcomes of this review are used to determine the scientific basis of diabetes nutrition applications to help advance, research, and development of future applications.

\section{Methods and Materials}

Google Play Store (Google Play Store (https://play.google. com) and the Apple iOS Store (https://itunes.apple.com) were used to find the applications with specific healthcare functionalities. The BlueStacks 4.1.14.1460 software was used to access these two stores. All applications were extracted and evaluated through the BlueStacks. According to the study requirements, the apps in a systematic evaluation on two operating systems were considered, independently. Sample searches were found in May 2018. The selected apps were reviewed in healthcare settings. This search was done on a set of free apps. Free programs were accurately and systematically considered for the domain of health and medical care. The input criteria for this study were: monitoring nutrition in diabetic patients, offering diet to diabetics, exercise and physical activity, and analyzing tools for activities and assessing the diet of diabetic patients. Initial selection and evaluation of the apps were carried out with review of the title and summary of the apps. Exclusions were those that were not specifically dedicated to diabetes for diabetics and health services and were not developed as a game or not accessible in English. The apps entered into this study were evaluated and information was collected to create 
observations through the text available in app stores and on the website of the applications (if present). The collected data were reviewed, collected, summarized, considered, and compared. Fig. 1 shows the flowchart and selection process.

\section{Application Analysis Tool}

Applications were studied, considered and analyzed (see Table 1 - Measuring tools for apps). The table was created with the factors used in the present and other studies. ${ }^{3,17,18}$ Table 2 shows the general criteria chosen for the study. Primary assessment criteria were administrated based on the identified and released apps in the Google Android market and the iOS. Then, the selected apps were determined based on inclusion criteria as the key factors. At first, apps related to diabetic patients were identified, then apps were limited to nutrition applications for diabetic patients. Finally, apps including the interactive features for analyzing data and interaction with the patients were the final goal. The study population of app users in this research involved patients with various types of diabetes, those affiliated with diabetic patients, such as family, doctors, nutritionists, and non-patients using the healthcare applications.

\section{Results}

This review is now for considering the features of the available apps on the mobile platform for consulting, educating, and nurturing diabetic patients. By using all inclusion criteria, finally 20 apps in Google Play and 2 applications in Apple iTunes were considered. Exclusion criteria for both app stores were games, non-English language, and duplicate applications. Most apps in the investigation outcomes provided one of the following services: cooking recipes for diabetics, nutritional information for patients, patient health records, self-care information, alert and reminders for insulin injections, weight control, and daily

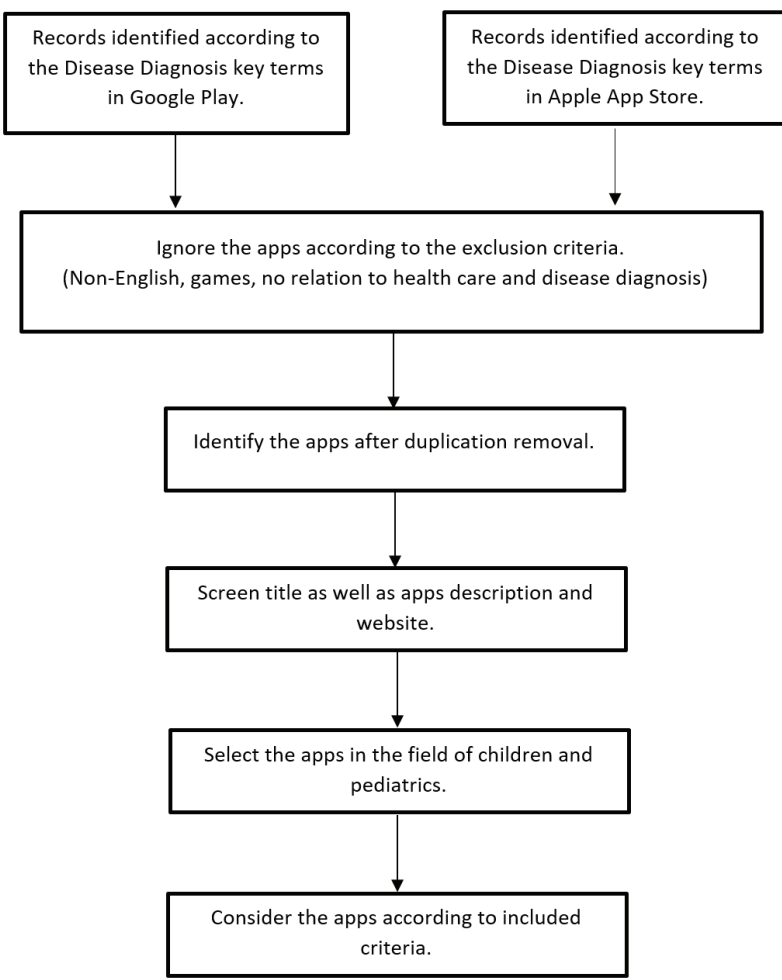

Fig. 1 Flowchart of systematic investigation process.

\begin{tabular}{lll}
\hline \multicolumn{2}{l}{ Table 1. Measurment tools. } \\
\hline $\begin{array}{c}\text { Factors for diabetes- } \\
\text { related apps in the } \\
\text { initial search }\end{array}$ & $\begin{array}{c}\text { Selected general } \\
\text { criteria for diabetes- } \\
\text { related apps }\end{array}$ & $\begin{array}{l}\text { Special criteria for } \\
\text { diabetes-related } \\
\text { apps }\end{array}$ \\
\hline $\begin{array}{l}\text { Insulin } \\
\text { Communication }\end{array}$ & $\begin{array}{l}\text { Nutrition plans } \\
\text { Interactive } \\
\text { data analysis } \\
\text { environment }\end{array}$ \\
$\begin{array}{l}\text { Diet } \\
\text { Body activity }\end{array}$ & Blood pressure \\
$\begin{array}{l}\text { Weight } \\
\text { Blood pressure }\end{array}$ & Body mass control \\
Personal health record & & \\
Education & & \\
Social media & & \\
Decision support & Weight & \\
Alternative medicine & & \\
Conference & & \\
Calculator & & \\
Monitoring/export and & &
\end{tabular}

activity control for patients. Fig. 2 shows the diagram of the screening process of this study. The results showed two hundred and fifty apps in Android market and twenty three apps in the iOS stores appeared in the review results (Fig. 2). To filter more search results, non-English programs, games, and unrelated apps were removed in further evaluations. The programs that were left were considered for review based on inclusion factors. We found that among the selected programs, a lot of the apps were designed to control insulin, weight control, selfcare in patients, and a list of dietary orders. Therefore, these apps were not included in the entry criteria such as dietary control, education, blood pressure control, and weight control, according to the status of diabetic patients.

The results showed that 20 apps covered the general criteria, and of these, 10 apps with special criteria in Google Play have been equipped. Table 2 presents the apps with initial and secondary criteria. In addition, Table 2 shows that there were not apps including all general and special criteria of this study in the iOS Apple store. Out of the 23 search apps with iOS, only 2 apps were found with the general criteria, while none were eligible according to special criteria in terms of the use of clinical interactive surface in nutrition for diabetic patients.

Fig. 2 shows the results of the inclusion criteria with evidence-based strategies and systematic evaluations to find the apps for diabetic patients. According to Fig. 2, approximately $29 \%$ of apps in both stores (79/273) met primary assessment criteria. Of these, $40 \%$ of apps (32/79) excluded more consideration due to lack in most criteria of this study. The remaining apps are described as follows: $7 \%$ of total apps (20/273) and $4 \%$ of the apps (10/273) met general criteria and all criteria (including general and special) in the Google Play Store, respectively. Only 2 apps met general criteria with the Apple iOS while none of them covered with special criteria according to the objectives of this study. 


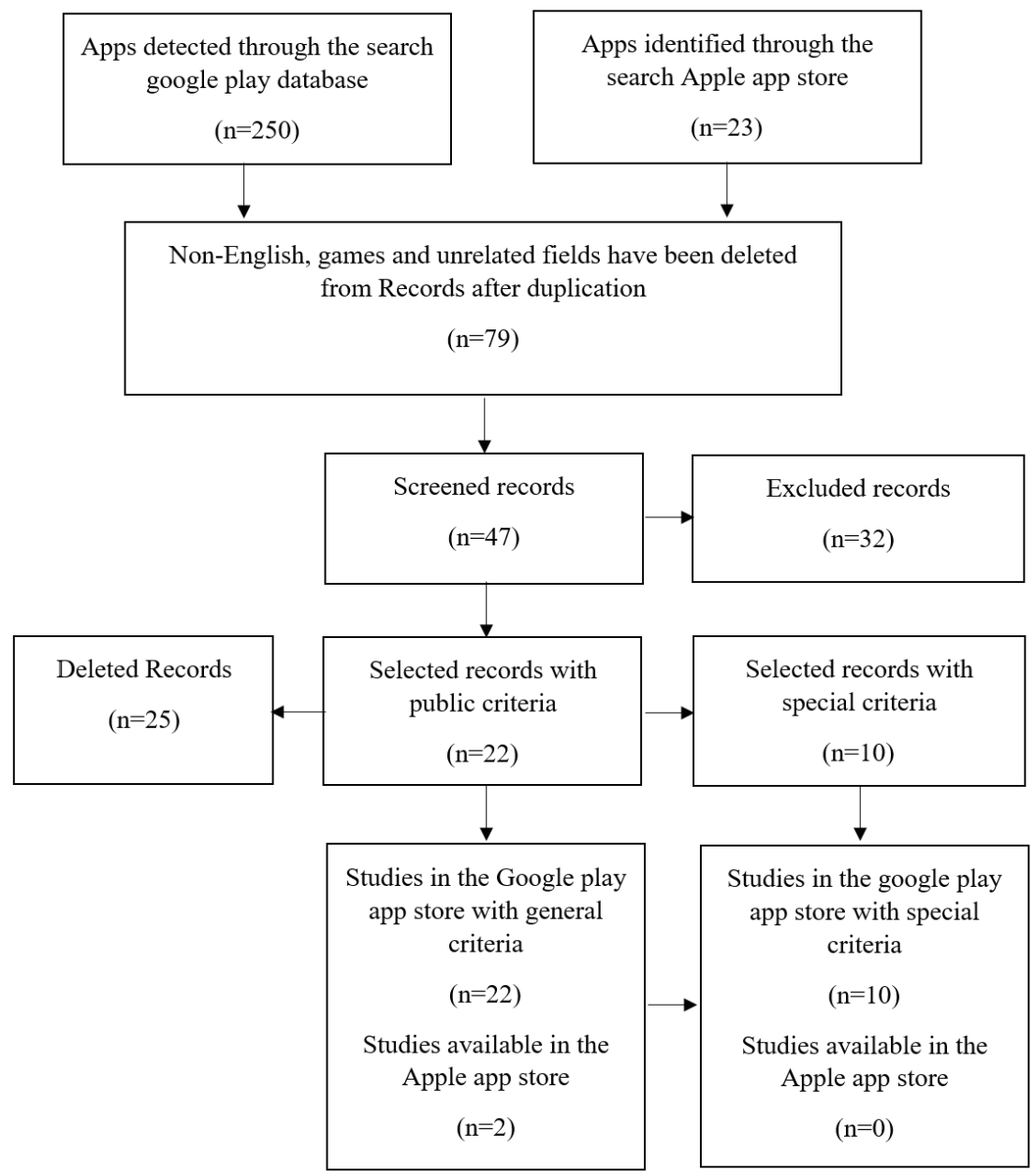

Fig. 2 Flowchart of the screeining process.

\section{Discussion}

Diabetes mellitus or diabetes is one of the most commonly diagnosed diseases in the world. Today, many efforts have been made to improve the diabetic patients care. In recent years, common approaches to using mobile technologies play a crucial role in medical care and patient self-management in a variety of diseases such as diabetes. Mobile health has emerged as the main part of the electronic health. The use of wireless technologies such as Bluetooth, Wi-max, Wi-Fi, Short Message Services, and data transmissions assist the users to access to the electronic health services. ${ }^{19}$ Mobile health apps collect and provide the healthcare data. These apps present the dynamic services for active participation of patients and providers in the healthcare and a new tool for improving health outcomes. ${ }^{20}$ This study presents an investigation of literature on a wide space of health apps for smart phones in the self-care and education for diabetics' patients. According to this review results, we found a lack of scientific information measuring the diagnostic value of health apps present in medical literature. The information about the diagnostic accuracy of currently available health apps on Apple's and Google's app stores is almost absent ${ }^{21}$. As a result, app users and healthcare professionals should be aware of the limitations when recommending specific programs. Most of the apps that appeared in this review results included a manual of dietary instructions for diabetic patients, only for patient monitoring, weight control, and glucose monitoring. The aim of this research was to systematically review the dietary apps for diabetics using the interactive environment for data and physical activity analyzing of patients. The methodologic consideration of the features in the apps showed the limitations of the iOS platform for developing the diabetic apps using the intelligent techniques. Blood Test Grapher and Blood Pressure Grapher were the only 2 apps with the iOS in which the diabetic features were used while lacking in terms of the interactive tools.

However, the Android Google supported several apps used for nutrition services including the intelligent techniques for analyzing of patients activities and control the diabetes effectively. Therefore, Android is a preferred platform for apps makers interested in gusing interactive tools in health and medical care applications.

\section{Conclusion}

As conclusion, today, due to increase in the incidence of diabetes as a worldwide disease, the need to address the applications created in this area has been considered. Diabetes education and self-care, especially food training, are the important 


\begin{tabular}{|c|c|c|c|c|c|c|}
\hline \multirow{2}{*}{ Applications } & \multirow{2}{*}{ Rating } & \multicolumn{2}{|c|}{ Platform } & \multirow{2}{*}{ Scope } & \multicolumn{2}{|c|}{ Inclusion criteria } \\
\hline & & Google Android & Apple App Store & & General & Special \\
\hline Sugar sense-diabetes app ${ }^{21}$ & 4.2 & $\checkmark$ & & Diabetic patient & $\checkmark$ & - \\
\hline Diabetic diet $\&$ symptoms of diabetes ${ }^{22}$ & 4.9 & $\checkmark$ & & $\begin{array}{l}\text { Diabetic patient and } \\
\text { normal patient }\end{array}$ & $\checkmark$ & $\checkmark$ \\
\hline Diabetic diet plan²3 & 4.3 & $\checkmark$ & & Diabetic patient & $\checkmark$ & $\checkmark$ \\
\hline Sugar free diet medicine reminder ${ }^{24}$ & 4.9 & $\checkmark$ & & Diabetic patient & $\checkmark$ & $\checkmark$ \\
\hline Diabetic calculator 25 & 4.1 & $\checkmark$ & & Type 1 diabetes & $\checkmark$ & - \\
\hline Diabetes treatment ${ }^{26}$ & 4.0 & $\checkmark$ & & Everyone & $\checkmark$ & - \\
\hline Low carb program 27 & 3.4 & $\checkmark$ & & $\begin{array}{l}\text { Type } 2 \text { diabetes and } \\
\text { prediabetes }\end{array}$ & $\checkmark$ & - \\
\hline How to reverse type 2 diabetes ${ }^{28}$ & 3.7 & $\checkmark$ & & Type 2 diabetes & $\checkmark$ & - \\
\hline Beat-diabetes care and management app ${ }^{29}$ & 4.7 & $\checkmark$ & & Diabetic patient & $\checkmark$ & $\checkmark$ \\
\hline SiDiary diabetes management ${ }^{30}$ & 4.4 & $\checkmark$ & & Diabetic patient & $\checkmark$ & \\
\hline One drop-diabetes management ${ }^{31}$ & 4.3 & $\checkmark$ & & Diabetic patient & $\checkmark$ & $\checkmark$ \\
\hline Diabetes pal ${ }^{32}$ & 3.8 & $\checkmark$ & & Diabetic patient & $\checkmark$ & \\
\hline Gather health family diabetes ${ }^{33}$ & 4.4 & $\checkmark$ & & Diabetic family members & $\checkmark$ & $\checkmark$ \\
\hline Is your diabetes under control $\left.\right|^{34}$ & 3.9 & $\checkmark$ & & $\begin{array}{l}\text { Diabetic patient and } \\
\text { everyone }\end{array}$ & $\checkmark$ & \\
\hline DiabetoMeter ${ }^{35}$ & 4.5 & $\checkmark$ & & $\begin{array}{l}\text { Diabetic patient and } \\
\text { diabetic family members }\end{array}$ & $\checkmark$ & $\checkmark$ \\
\hline Diabetes and symptoms ${ }^{36}$ & 4.1 & $\checkmark$ & & Diabetic patient & $\checkmark$ & \\
\hline Life in control diabetes coach ${ }^{37}$ & 4.5 & $\checkmark$ & & $\begin{array}{l}\text { Diabetic patient and } \\
\text { doctor }\end{array}$ & $\checkmark$ & $\checkmark$ \\
\hline Glucosio: Diabetes tracker ${ }^{38}$ & 4.2 & $\checkmark$ & & Diabetic patient & $\checkmark$ & \\
\hline Nutrition diabetes ${ }^{39}$ & 3.7 & $\checkmark$ & & $\begin{array}{l}\text { Diabetic patient and } \\
\text { doctor }\end{array}$ & $\checkmark$ & $\checkmark$ \\
\hline Diabetes mellitus from zero to hero ${ }^{40}$ & 4.9 & $\checkmark$ & & $\begin{array}{l}\text { Diabetic patient, doctor, } \\
\text { nurse, health worker }\end{array}$ & $\checkmark$ & $\checkmark$ \\
\hline Blood Test Grapher ${ }^{41}$ & No Ratings & & $\checkmark$ & Diabetic patient & $\checkmark$ & \\
\hline Blood Pressure Grapher ${ }^{42}$ & No Ratings & & $\checkmark$ & Diabetic patient & $\checkmark$ & \\
\hline
\end{tabular}

issues for the human healthcare. Given the widespread nature of this disease and the need for communities to educate and self-manage diabetes, there were a limited number of applications available that were designed and developed for nutrition with the patients' interactive tools for diabetic patients. In general, patients' interactive surface was used for a limited diabetes nutritional apps due to hardware and software constraints on mobile phones. The review results showed that Google is an acceptable platform for developers interested with inclusion criteria as well as in examining interactive apps and techniques in disease control, education and healthy lifestyle programs.

\section{Limitations}

This study was intended to find a review of the existing applications in the medical scope, in particular, the apps developed in the field of education and dietary orientation for diabetic patients. Therefore, the limitations of this study were: access to a large number of diabetes-related apps according to a wide range of criteria in the search program was a challenging issue, therefore, the criteria selected as the inclusion criteria were restricted in this study. Second, this investigation is meant to prepare a picture of applications existing in the medical industry instead of each specific specialization. Third, apps were considered in the field of medical care rather than apps with the scope of general healthcare. As a result, a big part of the applications were excluded from the final analysis.

\section{Acknowledgment}

Many thanks to research center of nutrition and metabolism at Ahvaz Jundishapur University of Medical Sciences, Iran to support this research. 


\section{Declaration of Conflicting Interest}

The author(s) declared there is not conflicts of interest in this study.

\section{References}

1. MM. Rahmati, S. Bakhshi. "Lifestyle and consuming pattern case study: Cellphone". J. Iran. Cult. Res. 8(4):119-42;2015.

2. M. Goodarzi, I. Ebrahimzadeh. "Impact of distance education via short message service of mobile phone on metabolic control of patients with type 2 diabetes mellitus in Karaj-Iran". Quart. Horizon Med. Sci.19(4):224$34: 2014$

3. HJ. Seabrook, JN. Stromer, C. Shevkenek, A. Bharwani, J. de Grood, WA. Ghali. "Medical applications: A database and characterization of apps in Apple iOS and Android platforms". BMC Res. Notes. 7(1):573;2014.

4. ASM. Mosa, I. Yoo, L. Sheets." A systematic review of healthcare applications for smartphones". BMC Med. Inform. Decision Making. 12(1):67;2012.

5. MNK. Boulos, S. Wheeler, C. Tavares, R. Jones. "How smartphones are changing the face of mobile and participatory healthcare: An overview, with example from eCAALYX". Biomed. Eng. Online. 10(1):24; 2011.

6. A. Karargyris, O. Karargyris, A. Pantelopoulos, editors. "DERMA/care: An advanced image-processing mobile application for monitoring skin cancer". Tools with Artificial Intelligence (ICTAI), 2012 IEEE 24th International Conference on; 2012: IEEE.

7. J. Rivera, A. McPherson, J. Hamilton, C. Birken, M. Coons, S. Iyer, et al." Mobile apps for weight management: a scoping review". JMIR mHealth and uHealth. 4(3);2016.

8. B. Martínez-Pérez, I. De La Torre-Díez, M. López-Coronado, J. HerrerosGonzález. "Mobile apps in cardiology". JMIR mHealth and uHealth. 1(2);2013.

9. CL. Ventola." Mobile devices and apps for health care professionals: uses and benefits". Pharm. Therap. 39(5):356;2014.

10. T. Dehling, F. Gao, S. Schneider, A. Sunyaev. “Exploring the far side of mobile health: information security and privacy of mobile health apps on iOS and Android". JMIR mHealth and uHealth. 3(1);2015.

11. E-J. Lee, Y-H. Kim, N. Kim, D-W. Kang." Deep into the brain: Artificial intelligence in stroke imaging". J. Stroke. 19(3):277; 2017

12. D. Shanthi, G. Sahoo, N. Saravanan." Designing an artificial neural network model for the prediction of thrombo-embolic stroke". Int. J. Biomet. Bioinform. (IJBB). 3(1):10-8;2009.

13. N. Mirderikvand, M. Naderan, A. Jamshidnezhad. "Accurate automatic localisation of lung nodules using Graph Cut and snakes algorithms". 2016 6th International Conference on Computer and Knowledge Engineering (ICCKE). 194-199; 2016.

14. L. Kabootarizadeh, A. Jamshidnezhad, Z. Koohmareh, A. " Differential Diagnosis of Iron-Deficiency Anemia from $\beta$-Thalassemia Trait Using an Intelligent Model in Comparison with Discriminant Indexes". Acta Inform Med. 27(2): 78-84; 2019

15. R. Altman. "Artificial intelligence (Al) systems for interpreting complex medical datasets". Clin. Pharmacol. Therap. 101(5):585-6;2017.

16. H. Vainio, F. Bianchini. "Weight control and physical activity": Iarc; 2002.

17. T. Chomutare, L. Fernandez-Luque, E. Årsand, G. Hartvigsen. "Features of mobile diabetes applications: Review of the literature and analysis of current applications compared against evidence-based guidelines". J. Med. Internet Res. 13(3);2011.

18. O. El-Gayar, P. Timsina, N. Nawar, W. Eid." Mobile applications for diabetes self-management: Status and potential. J. Diab. Sci. Technol". 7(1):247-62; 2013

19. R. Ershad Sarabi, F. Sadoughi, R. Jamshidi Orak, K. Bahaadinbeigy. "Role of mobile technology in Iran healthcare system: A review study". J. Health Biomed. Inform. 4(4):313-26;2018.

20. MHM. Jazayeri, A. Jamshidnezhad, "Top mobile applications in pediatrics and children's health: Assessment and intelligent analysis tools for a systematic investigation". MJMS 26(1):5-14; 2019

21. Buechi R, Faes L, Bachmann LM, Thiel MA, Bodmer NS, Schmid MK, et al. "Evidence assessing the diagnostic performance of medical smartphone apps: a systematic review and exploratory meta-analysis". BMJ open. 2017;7(12):e018280.
22. Lazer Apps Inc. Diabetic Diet \& Symptoms of diabetes. Google play [Download]. Available at: https://play.google.com/store/apps/ details?id=com.lazerapps.android.diabetesgo\&hl=en_US.

23. Gato Apps. Diabetic Diet Plan. Google play [Download]. Available at https:// play.google.com/store/apps/details?id=com.gatoapps.diabeticdiet\&hl=en_ US.

24. Manafi Group. Sugar Free Diet Medicine Reminder. Google play [Download]. Available at https://play.google.com/store/apps/details?id=sugarfree.diet. medicinereminder\&hl=en_US.

25. Jake2701. Diabetic Calculator. Google play [Download]. Available at https:// play.google.com/store/apps/details?id=mine.mydiabeticcalculator\&hl=en_ US.

26. Xtell Technologies. Diabetes Treatment. Google play [Download]. Available at https://play.google.com/store/apps/details?id=com.xtelltechnologies. diabetestreatment.

27. Diabetes Digital Media. Low Carb Program. Google play [Download] Available at https://play.google.com/store/apps/details?id=uk.co.diabetes lowcarb\&hl=en.

28. HealthSensei101. How To Reverse Type 2 Diabetes. Google play [Download]. Available at https://play.google.com/store/apps/details?id=com. diabetes60system.type2diabetes\&hl=en.

29. BeatO. Beat0-Diabetes Care and Management App. Google play [Download]. Available at https://play.google.com/store/apps/ details?id=com.healtharx.beato\&hl=en

30. SINOVO GmbH \& Co. KG. SiDiary Diabetes Management. Google play [Download]. Available at https://play.google.com/store/apps/ details?id=com.sidiary.app\&hl=en.

31. One Drop. One Drop- Diabetes Management. Google play [Download]. Available at https://play.google.com/store/apps/details?id=today.onedrop. android\&hl=en.

32. Telcare LLC. Diabetes Pal. Google play [Download]. Available at https://play. google.com/store/apps/details?id=com.telcare.android.client\&hl=en.

33. Gather Health. Gather Health Family Diabetes. Google play [Download]. Available at https://play.google.com/store/apps/details?id=com. gatherhealth.gatherdm\&hl=en.

34. Twayesh Projects. Is your Diabetes under control. Google play [Download] Available at https://play.google.com/store/apps/details?id=anace.com. audiobooks.diabetes\&hl=en_US.

35. Ghrian Technologies Pvt. Ltd. DiabetoMeter. Google play [Download]. Available at https://play.google.com/store/apps/details?id=com. ghriantech.diabetometer\&hl=en_US.

36. Appz Inventors. Diabetes and Symptoms. Google play [Download] Available at https://play.google.com/store/apps/details?id=com.appz. Diabetes\&hl=en.

37. Life in Control. Life in Control Diabetes Coach. Google play [Download]. Available at https://play.google.com/store/apps/details?id=com. lifeincontrol\&hl=en

38. Glucosio. Glucosio: Diabetes Tracker. Google play [Download]. Available at https://play.google.com/store/apps/details?id=org.glucosio. android\&hl=en_US.

39. Built by Doctors World Ltd. Nutrition Diabetes. Google play [Download]. Available at https://play.google.com/store/apps/details?id=com. builtbydoctors.ntdiabetes\&hl=en

40. Utopia production. Diabetes mellitus(DM) from zero to hero. Google play [Download]. Available at https://play.google.com/store/apps/ details?id=com.Utopia.Diabetes777\&hl=en.

41. Ai Imai. Blood Test Grapher. iTunes [Download]. Available at https://itunes. apple.com/us/developer/ai-imai/id1133573418?mt=8.

42. Ai Imai. Blood Pressure Grapher. iTunes [Download]. Available at https://itunes.apple.com/ro/app/blood-pressure-grapher/ id1171409283? $\mathrm{mt}=8$

This work is licensed under a Creative Commons Attribution-NonCommercial 3.0 Unported License which allows users to read, copy, distribute and make derivative works for non-commercial purposes from the material, as long as the author of the original work is cited properly. 